\section{Avaliação da microestrutura e das propriedades magnéticas de ferrita de cobre dopada com chumbo sinterizada com fase líquida}

\author{
Evaluation of microstructure and magnetic \\ properties of copper ferrite doped with \\ lead sintered with liquid phase
}

Vander Alkmin dos Santos Ribeiro ${ }^{1}$, Amanda Carvalho Pereira ${ }^{1}$, Adhimar Flávio Oliveira ${ }^{1}$, Claudiney de Sales Pereira Mendonça ${ }^{1}$, Manoel Ribeiro da Silva ${ }^{1}$

\footnotetext{
${ }^{1}$ Universidade Federal de Itajubá, Av. BPS, 1303, bairro Pinheirinho, Itajubá - MG, Caixa Postal 50 CEP: 37500-903. e-mail: vanderalkmin@gmail.com, amandinhafar@yahoo.com.br, adhimarflavio@unifei.edu.br, sales.claudiney21@gmail.com,mrsilva@unifei.edu.br
}

\section{RESUMO}

Este artigo apresenta a obtenção de uma ferrita de chumbo e cobre com a estrutura do espinélio, a caracterização da sua microestrutura e a determinação das suas propriedades magnéticas. A ferrita de cobre e chumbo é um material com baixa coercividade magnética e alta resistividade elétrica. Amostras com estequiometria $\mathrm{Pb}_{\mathrm{x}} \mathrm{Cu}_{1-\mathrm{x}} \mathrm{Fe}_{2} \mathrm{O}_{4},(\mathrm{x}=0,00 ; 0,05 ; 0,10 ; 0,15$ e 0,20) foram sintetizadas pelo processo de metalurgia do pó e sinterizadas a $1000{ }^{\circ} \mathrm{C}$ por $6 \mathrm{~h}$. Os resultados mostram que a amostra com $\mathrm{x}=0,00$ é formada pela estrutura do espinélio cúbico e x variando de 0,05 a 0,20 são formadas exclusivamente pela estrutura do espinélio tetragonal. As curvas de histerese mostram características de materiais magnéticos moles e a magnetização de saturação e temperatura de Curie diminuíram com a concentração de chumbo, no entanto observou-se o aumento do campo coercivo.

Palavras-chave: Ferrita de chumbo, Microestrutura, Fase Líquida, Propriedades magnéticas, Efeito JahnTeller.

\section{ABSTRACT}

This paper presents to obtain a lead and copper ferrite with spinel structure, characterizing the microstructure and the determination of their magnetic properties. The copper and lead ferrite is a material with low magnetic coercivity, high electrical resistivity. Samples with stoichiometry $\mathrm{Pb}_{\mathrm{x}} \mathrm{Cu}_{1-\mathrm{x}} \mathrm{Fe}_{2} \mathrm{O}_{4}$ with $(\mathrm{x}=0.00 ; 0.05 ; 0.10$; 0.15 e 0.20 ) were prepared by powder metallurgy processes and sintered at $1000{ }^{\circ} \mathrm{C}$ for 6 hours. The results show that sample with $\mathrm{x}=0.00$ is formed exclusively by the cubic spinel structure and samples with $\mathrm{x}$ between 0.05 to 0.20 are formed exclusively by the tetragonal spinel structure hysteresis loops show the characteristics of a soft magnetic material and the saturation magnetization and Curie temperature decreased with the lead concentration, however, noted that the increase in the coercive field.

Keywords: Lead ferrites, Microestruture, Liquid Phase, Magnetic properties, Jahn-Teller Effect.

\section{INTRODUÇÃO}

A ferrita de cobre dopada com chumbo do tipo espinélio é um material com baixa coercividade magnética e remanescência, alta temperatura de Curie, alto valor de resistividade elétrica e alta densidade. Este material desempenha papel de grande importância no campo científico devido às suas várias aplicações tecnológicas, tais como transformadores de potência e de pulso, transformadores para distribuição de energia, transformadores de banda larga e baixa potência. 
As ferritas do tipo espinélio possuem fórmula química $\mathrm{MFe}_{2} \mathrm{O}_{4}$, onde $\mathrm{M}$ é um íon divalente. Os íons de oxigênios formam uma estrutura cúbica simples compacta com 64 interstícios tetraédricos e 32 interstícios octaédricos, no qual 8 interstícios tetraédricos e 16 interstícios octaédricos são ocupados por cátions [1,2]. Um cátion divalente tende a ocupar os sítios tetraédricos para a formação de uma estrutura de espinélio normal, se estes cátions ocuparem os sítios octaédricos formam uma estrutura de espinélio inversa [1].

Quando estas ferritas são dopadas com cobre ocorre uma distorção na rede cristalina, ou seja, a célula unitária do espinélio sofre um discreto crescimento em uma das arestas, devido a inclusão de certa quantidade de cobre. O crescimento em uma das arestas muda a simetria do espinélio cúbico para tetragonal, sendo este efeito denominado efeito Jahn-Teller $[3,4,5,6,7,8]$. Neste caso a mudança da estrutura ocorre porque o $\mathrm{Cu}^{2+}$ ocupa os interstícios tetraédricos ou octaédricos, enquanto que o $\mathrm{Fe}^{3+}$ passa a ocupar os interstícios tetraédricos $[9,10,11,12]$.

Sempre deseja-se obter ferritas de qualidade que possuam alta densidade e um grande tamanho de grão. As propriedades magnéticas das ferritas são dependentes da composição química, do tamanho de grão ou da estrutura cristalina. Trabalhos anteriores mostram que o tamanho de grão é um dos parâmetros mais importantes que afetam as propriedades magnéticas [13]. Por exemplo, a elevada permeabilidade magnética inicial pode ser alcançada apenas em amostras com grande tamanho de grão e com a baixa porosidade residual restrita no contorno de grão [13,14].

Para a obtenção de ferritas com alta densidade e grande tamanho de grão, a sinterização via fase líquida aliada a dopagem com PbO é uma boa escolha. A fase líquida atrai os grãos em conjunto (devido à força de capilaridade) promovendo um forte e rápido rearranjo dos grãos que leva à maior densificação e crescimento do grão. Entretanto, a sinterização por fase líquida deve ser cuidadosamente controlada, pois pode acarretar ao crescimento anormal do grão, com consequente aumento da porosidade e degradação das propriedades magnéticas [15,16,17]. A dopagem com PbO auxília o crescimento do grão durante a sinterização, mesmo em baixos níveis de dopagem trazendo benefícios para a densificação, consequentemente diminui a porosidade do material e possui baixo ponto de fusão $\left(\sim 880^{\circ} \mathrm{C}\right)$, portanto, fornece um excelente meio de sinterização via fase líquida $[18,19]$.

MIRZAEE [13] investigou o efeito da dopagem de $\mathrm{PbO}$ (0,00 a 3,20 \% em peso, com intervalos de $0,40 \%$ ) na ferrita de Ni-Zn sinterizadas a $1300{ }^{\circ} \mathrm{C}$ por $2 \mathrm{~h}$. Os resultados mostraram que a densidade aumentou de 5,15 g/ $\mathrm{cm}^{3}$, nas amostras isentas de $\mathrm{PbO}$, para 5,30 g/ $/ \mathrm{cm}^{3}$ nas amostras contendo 1,60 \% de PbO, para $4,98 \mathrm{~g} / \mathrm{cm}^{3}$ nas amostras com 3,20 \% de PbO. O aumento da densidade ocorreu devido à formação de fase líquida de $\mathrm{PbO}$ entre grãos da ferrita, por outro lado, o aumento excessivo da espessura da camada da fase líquida resultou na redução da taxa de difusão e, consequentemente, houve uma redução na densidade. A variação da percentagem de $\mathrm{PbO}$ também causou variação no tamanho de grão, que aumentou de 1,8 $\mu \mathrm{m}$ na amostra isenta de $\mathrm{PbO}$, para 17,1 $\mu \mathrm{m}$ nas amostras contendo 2,40 \% de $\mathrm{PbO}$ para 6,4 $\mu \mathrm{m}$ nas amostras contendo 3,20 \% de $\mathrm{PbO}$ [13]. A variação do tamanho de grão é evidenciada em três faixas de dopagem distintas.

A primeira, em concentrações de 0,00 a $0,40 \%$ em peso ocorre a difusão na ausência ou pequenas quantidades da fase líquida nos contornos de grão. Esta região é caracterizada por pequenos tamanhos de grão. Em concentrações com 0,40 a 2,40 \% em peso ocorre a difusão através da fase líquida rica em $\mathrm{PbO}$. Esta faixa de concentação é caracterizada pelo rápido crescimento de grãos e consideravel diminuição da energia de ativação. Em concentrações maiores que 2,40 \% em peso ocorre o aumento da espessura da camada da fase líquida rica em $\mathrm{PbO}$ diminuindo a densidade aparente [13]. Com relação à temperatura de Curie observou-se que a variação na percentagem de $\mathrm{PbO}$ aumentou de $247^{\circ} \mathrm{C}$ para a ferrita isenta de chumbo para $252{ }^{\circ} \mathrm{C}$ para a ferrita contendo $1,6 \%$ de $\mathrm{PbO}, 233{ }^{\circ} \mathrm{C}$ para a amostra contendo 3,2 \%. Também observou-se que a magnetização de saturação diminuiu de 78,3 emu/g para a ferrita isenta de $\mathrm{PbO}$ para 71,2 emu/g para a ferrita com 3,20 \% em peso de $\mathrm{PbO}$ [13].

ULLAH et al. [19] investigou o efeito da dopagem de $\mathrm{Pb}^{2+}$ na ferrita com estequiometria $\mathrm{Sr}_{1-}$ ${ }_{\mathrm{x}} \mathrm{Pb}_{\mathrm{x}} \mathrm{Fe}_{12} \mathrm{O}_{19}(\mathrm{x}=0,00 ; 0,05 ; 0,10 ; 0,15$ e 0,20$)$ sinterizadas a $800{ }^{\circ} \mathrm{C}$ por $2 \mathrm{~h}$. Os resultados mostraram que a dopagem com $\mathrm{PbO}$ na ferrita de estrôncio afetaram consideravelmente a magnetização de saturação, campo coercivo e remanência. A magnetização de saturação diminuiu de $(45,80 \pm 0,75)$ emu/g para a ferrita isenta de chumbo $(x=0,00)$ para $(24,60 \pm 0,60)$ emu/g nas ferritas com estequiometria $x=0,20$ e a remanência diminuiu de $(22,80 \pm 0,60)$ para a ferrita isenta de chumbo para $(14,80 \pm 0,40)$ emu/g nas ferritas com estequiometria $\mathrm{x}=0,20$ [19]. A coercividade aumentou de $(2280 \pm 40)$ Oe para a ferrita isenta de chumbo $(\mathrm{x}=0,00)$ para $(3820 \pm 40)$ Oe nas ferritas com estequiometria $\mathrm{x}=0,20$. A diminuição da magnetização de saturação ocorreu devido à substituição de um material paramagnético $\left(\mathrm{Sr}^{2+}\right)$ por um diamagnético $\left(\mathrm{Pb}^{2+}\right)$ [19].

HAQ e ANIS-UR-REHMAN [20] investigou o efeito da dopagem de $\mathrm{PbO}$ na ferrita com estequiometria $\mathrm{Ba}_{1-\mathrm{x}} \mathrm{Pb}_{\mathrm{x}} \mathrm{Fe}_{12} \mathrm{O}_{19}(\mathrm{x}=0,0 ; 0,20 ; 0,40 ; 0,60 ; 0,80$ e 1,00$)$ sinterizadas a $965{ }^{\circ} \mathrm{C}$ por $3 \mathrm{~h}$. Os resultados mos- 
traram que o campo coercivo aumentou de 5,34 Oe para a ferrita isenta de chumbo $(\mathrm{x}=0,00)$ para 5,39 Oe para a ferrita com estequiometria $x=0,20$ [20]. A partir desta concentração houve uma diminuição de 5,39 Oe das amostras com estequiometria $\mathrm{x}=0,20$ para 2,72 Oe das amostras com estequiometrias com $\mathrm{x}=1,00$. O campo coercivo diminuiu devido ao movimento limitado nas paredes dos domínios e aumento do tamanho de grão [20].

Este artigo apresenta a obtenção de uma ferrita de chumbo e cobre com a estrutura do espinélio de estequiometria $\mathrm{Pb}_{\mathrm{x}} \mathrm{Cu}_{1-\mathrm{x}} \mathrm{Fe}_{2} \mathrm{O}_{4}(\mathrm{x}=0,00 ; 0,05 ; 0,10 ; 0,15 ; 0,20)$ preparadas pelo processo de metalurgia do pó, a caracterização microestrutural e magnética das amostras.

\section{MATERIAIS E MÉTODOS}

Amostras com estequiometria $\mathrm{Pb}_{\mathrm{x}} \mathrm{Cu}_{1-\mathrm{x}} \mathrm{Fe}_{2} \mathrm{O}_{4}(\mathrm{x}=0,00 ; 0,05 ; 0,10 ; 0,15 ; 0,20)$ foram preparadas pelo processo de metalurgia do pó utilizando $\mathrm{CuO}, \mathrm{Fe}_{2} \mathrm{O}_{3}$ e $\mathrm{PbO}$ com pureza mínima de 99,9 \% em peso. As amostras foram compactadas em uma prensa uniaxial e sinterizadas a $1000{ }^{\circ} \mathrm{C} / 6 \mathrm{~h}$ em atmosfera ambiente.

A caracterização estrutural foi realizada pelas técnicas de difração de raios-X. A difração de raios-x foi realizada utilizando-se um difratômetro da marca Panalytical modelo Xpert Pro MPD com radiação CuK ${ }_{\alpha}$, ângulo de varredura de $15^{\circ}$ a $80^{\circ}$, passo de $0,02^{\circ} \mathrm{S}^{-1}$ e tempo de contagem de 2 s por ponto. $\mathrm{O}$ refinamento das estruturas cristalinas foram realizados utilizando o software FullProff.

Os parâmetros magnéticos foram determinados através das curvas de histerese magnética obtidas através de um magnetômetro de amostra vibrante comum (VSM) da marca EG\&G-Princeton Applied Research, modelo 7404, campo aplicado até $14 \mathrm{kG}$. Os resultados do momento magnético apresentam uma incerteza menor que $1 \%$.

A temperatura de Curie foi identificada aplicando-se um campo magnético sobre a amostra sinterizada em um aparelho Analisador Termogravimétrico da marca Shimadzu, modelo TGA-50, entre a temperatura ambiente e $1000{ }^{\circ} \mathrm{C}$ com uma taxa de aquecimento de $10{ }^{\circ} \mathrm{C} / \mathrm{min}$. A temperatura de curie foi calculada através da análise das curvas de magnetização em função da temperatura através do efeito Hopkinson. Pela análise da derivada $\mathrm{dM} / \mathrm{dT}$ temos indícios da temperatura de Curie do material. Os resultados da magnetização apresentam uma incerteza menor que $1 \%$.

As micrografias foram obtidas utilizando-se um equipamento da marca Zeiss, modelo EVO MA15. A determinação do tamanho médio de grão foi feita utilizando-se o programa de processamento digital "Image $J$ ” realizada em micrografia conforme a norma ASTM E 1382-97 [21]. A partir das imagens, fez-se a medida do diâmetro de Feret, que indica a distância entre duas retas paralelas que tangenciam o objeto, excluindo os grãos dispostos na borda da imagem. As imagens foram analisadas atingindo a contagem média de 1000 grãos por amostra e a incerteza foi calculada através do limite de erro estatístico [22].

Os valores da densidade a verde das amostras não sinterizadas foram obtidos pelo método massa por volume e a densidade aparente das amostras sinterizadas foram determinadas pelo método de imersão utilizando o princípio de Arquimedes (norma ABNT-NBR 6620) [6,23,24,25].

\section{RESULTADOS E DISCUSSÕES}

A Figura 1 mostra os difratogramas de raios-x das amostras sinterizadas a $1000{ }^{\circ} \mathrm{C}$ por $6 \mathrm{~h}$ com diferentes estequiometrias de $x=0,00$ a $x=0,20$. Através do difratograma observou-se para a amostra isenta de chumbo $(x=0,00)$ que as fases são formadas exclusivamente pela fase ferrita com estrutura de espinélio com simetria cúbica (Ficha padrão JCPDS 01-077-0010) e para as amostras com estequiometria contendo $x=0,05$ a $x$ $=0,20$ observou-se que as fases são formadas exclusivamente pela fase ferrita com estrutura de espinélio com simetria tetragonal (Ficha padrão JCPDS 34-0425). A transformação do espinélio cúbico para tetragonal é encontrado em concentrações de cobre com $x=0,00$ para $x=0,30$ [26]. No nosso caso, as amostras com estequiometria contendo $x=0,05$ a $x=0,20$ há indícios do efeito Jahn-Teller. 


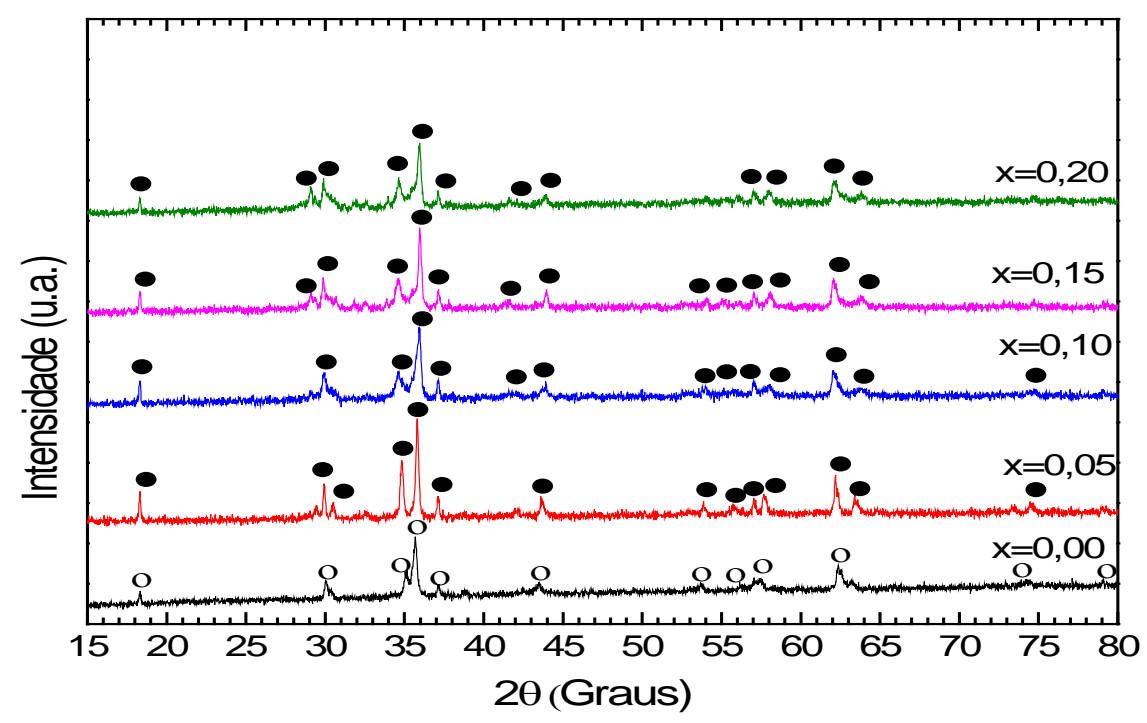

Figura 1: Difratogramas para a ferrita de $\mathrm{Pb}_{\mathrm{x}} \mathrm{Cu}_{(1-\mathrm{x})} \mathrm{Fe}_{2} \mathrm{O}_{4}$ de $\mathrm{x}=0,00$ a x=0,20. (o) Cúbica e (•) tetragonal.

Através do refinamento da estrutura cristalina observou-se uma variação dos parâmetros de rede nas amostras sinterizadas a $1000^{\circ} \mathrm{C}$ por $6 \mathrm{~h}$, conforme apresentado na Tabela 1. Os baixos valores de $S(<2)$ são indicativos de uma boa precisão para os resultados obtidos no refinamento.

Tabela 1: Parâmetros de rede para a estrutura do espinélio para amostras sinterizadas a $1000{ }^{\circ} \mathrm{C} / 6 \mathrm{~h}$.

\begin{tabular}{lcccccc}
\hline AMOSTRAS & $\mathbf{a}(\AA)$ & $\mathbf{b}(\AA)$ & $\mathbf{c}(\AA)$ & Rwp (\%) & Rexp (\%) & $\begin{array}{c}\text { FATOR DE } \\
\text { QUALIDADE (S) }\end{array}$ \\
\hline $\mathrm{X}=0,00$ & 8,41 & 8,41 & 8,41 & 3,71 & 3,23 & 1,14 \\
$\mathrm{X}=0,05$ & 5,87 & 5,87 & 8,63 & 3,43 & 3,41 & 1,00 \\
$\mathrm{X}=0,10$ & 5,87 & 5,87 & 8,63 & 3,60 & 3,57 & 1,00 \\
$\mathrm{X}=0,15$ & 5,84 & 5,84 & 8,68 & 3,76 & 3,57 & 1,05 \\
$\mathrm{X}=0,20$ & 5,82 & 5,82 & 8,63 & 3,72 & 3,69 & 1,00 \\
& & & & & & \\
\hline
\end{tabular}

A Figura 2 mostra as curvas de histerese em função do campo aplicado a temperatura ambiente das amostras sinterizadas a $1000^{\circ} \mathrm{C}$ por $6 \mathrm{~h}$ com estequiometria contendo $\mathrm{x}=0,00$ a $\mathrm{x}=0,20$. As curvas de histerese da amostra isenta de chumbo $(\mathrm{x}=0,00)$ a amostra com estequiometria contendo $\mathrm{x}=0,20$ possuem características de um material magnético mole, comportamento que foi atribuído aos valores menores que 1000 Oe do campo coercivo (Hc) e área interna estreita do ciclo de histerese. 


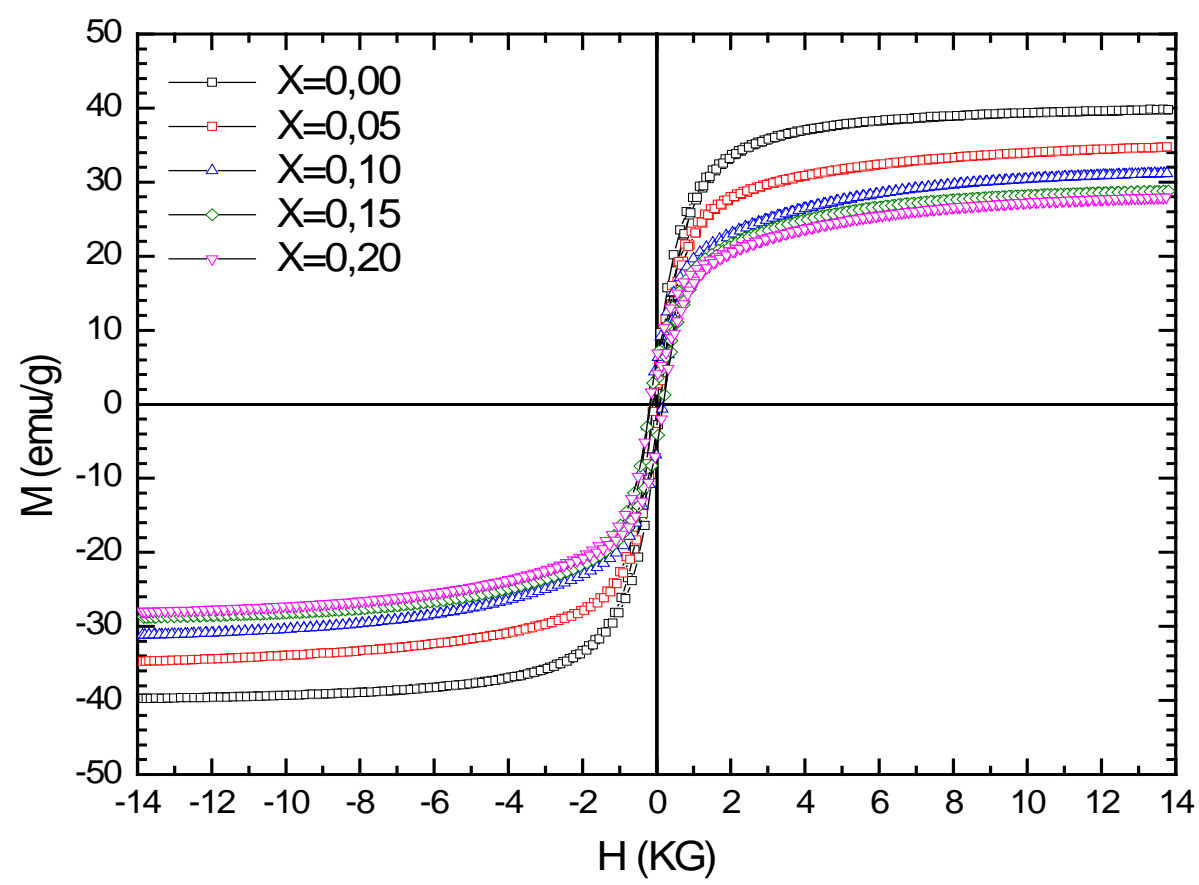

Figura 2: Ciclo de histerese para as amostras sinterizadas a $1000^{\circ} \mathrm{C} / 6 \mathrm{~h}$.

A tabela 2 mostra os resultados da magnetização de saturação $(\mathrm{Ms})$, campo coercivo $\left(\mathrm{H}_{\mathrm{C}}\right)$ e magnetização remanente $\left(\mathrm{M}_{\mathrm{R}}\right)$ para as amostras sinterizadas a $1000^{\circ} \mathrm{C}$ por $6 \mathrm{~h}$. A magnetização de saturação decresceu de 39,73 emu/g para a amostra isenta de chumbo $(\mathrm{x}=0,00)$ para 27,99 emu/g para a amostra com estequiometria contendo $x=0,20$ e a magnetização remanente decresceu de 6,04 emu/g para a amostra isenta de chumbo $(\mathrm{x}=0,00)$ para 4,60 emu/g para a amostra com estequiometria $\mathrm{x}=0,20$. Há indícios que o decréscimo da magnetização de saturação e da magnetização remanente com o acréscimo de $\mathrm{PbO}$ ocorreu devido ao aumento da porosidade e a substituição do $\mathrm{Pb}^{2+}$ (diamagnético) pelo $\mathrm{Cu}^{2+}$ (paramagnético) $[27,28]$. Observase também que o campo coercivo aumentou de 74,31 $\mathrm{G}$ na amostra isenta de chumbo $(\mathrm{x}=0,00)$ para 181,91 $\mathrm{G}$ na amostra com estequiometria contendo $\mathrm{x}=0,20$. De acordo com a teoria de Stoner-Wohlfarth, o campo coercivo dos materiais magnéticos está relacionado com a constante anisotrópica magneto-cristalina e a magnetização de saturação [19]. A tabela 2 observa-se que a magnetização de saturação diminuiu e o campo coercivo aumentou com a concentração de chumbo. Quando dopamos um material com PbO, a anisotropia magneto-cristalina é afetada e consequentemente pode aumentar seu campo coercivo e diminuir a magnetização de saturação [19].

Tabela 2: Valores da magnetização de saturação $\left(M_{s}\right)$, coercividade $\left(H_{c}\right)$, Magnetização Remanente $\left(M_{r}\right)$ para amostras sinterizadas a $1000{ }^{\circ} \mathrm{C}$ por $6 \mathrm{~h}$.

\begin{tabular}{c|c|c|c}
\hline AMOSTRAS & $\mathbf{M}_{\mathbf{s}}(\mathbf{e m u} / \mathbf{g})$ & $\mathbf{H}_{\mathbf{C}} \mathbf{( G )}$ & $\mathbf{M}_{\mathbf{R}}(\mathbf{e m u} / \mathbf{g})$ \\
\hline$x=0,00$ & 39,73 & 74,31 & 6,04 \\
\hline$x=0,05$ & 34,70 & 112,14 & 5,97 \\
\hline$x=0,10$ & 31,13 & 142,68 & 5,76 \\
\hline$x=0,15$ & 28,75 & 165,94 & 5,26 \\
\hline$x=0,20$ & 27,99 & 181,91 & 4,60 \\
\hline
\end{tabular}

A Figura 3 mostra as curvas de magnetização em função da temperatura para as amostras de estequiometria contendo $\mathrm{x}=0,00$ a $\mathrm{x}=0,20$ sinterizadas a $1000^{\circ} \mathrm{C}$ para a identificação da temperatura de Curie.

A medida que as amostras são aquecidas na presença de um campo magnético verifica-se em uma faixa de temperatura uma perda de magnetização acentuada denominada de efeito Hopkinson. O efeito Hopkin- 
son caracteriza-se por um pico na curva magnetização em função da temperatura próximo a Temperatura de Curie, e é baseado no movimento das paredes de domínio.

Durante o aquecimento, a mobilidade das paredes de domínio aumenta na proximidade da temperatura de Curie aumentando a componente da magnetização na direção do campo externo [29]. Para a amostra isenta de chumbo $(\mathrm{x}=0,00)$ observa-se dois comportamentos distintos: (i) a temperatura de $446,58{ }^{\circ} \mathrm{C}$ há indícios de transformação de fase da estrutura cúbica para tetragonal devido a distorção Jahn-Teller [5,30]. BALAGUROV et al. [30] mostrou que a transição estrutural da fase cúbica para a fase tetragonal na amostra isenta de chumbo $(\mathrm{x}=0,00)$ ocorre em temperatura aproximada de $440,00{ }^{\circ} \mathrm{C}$ e (ii) a temperatura de $493,00{ }^{\circ} \mathrm{C}$ há indícios da transição de Curie. SHAHIDA AKHTER et al. [31] investigou a ferrita isenta de chumbo ( $\mathrm{x}=$ $0,00)$ e identificou um valor da temperatura de Curie de aproximadamente $470{ }^{\circ} \mathrm{C}$.

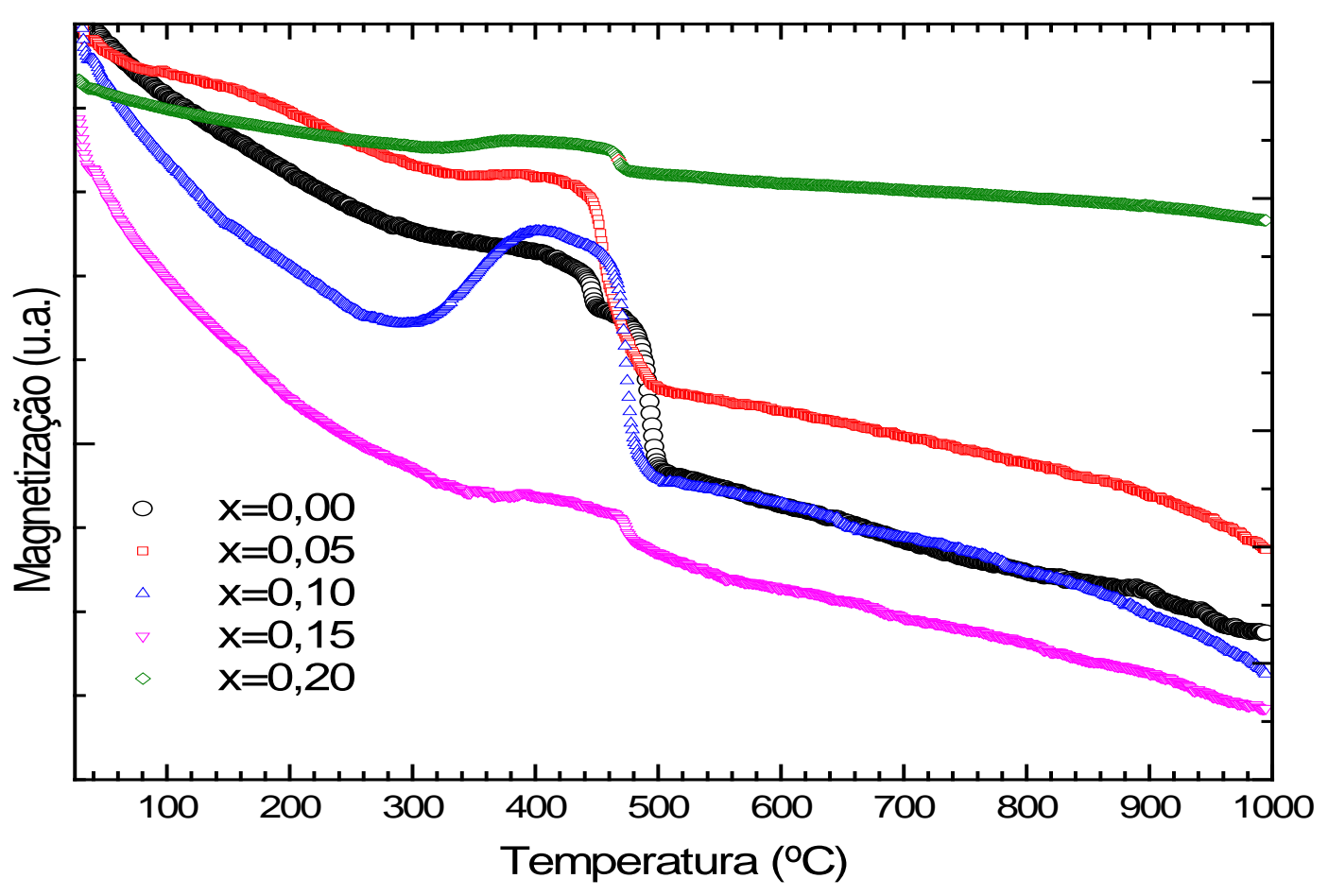

Figura 3: Curvas de magnetização em função da temperatura para as amostras sinterizadas a 1000 º $/ 6 \mathrm{~h}$.

A Tabela 3 mostra que a temperatura de Curie das amostras diminuiu de 493,00 ${ }^{\circ} \mathrm{C}$ na amostra isenta de chumbo $(\mathrm{x}=0,00)$ para $469,00^{\circ} \mathrm{C}$ na amostra com estequiometria contendo $\mathrm{x}=0,20$. A temperatura de Curie diminuiu com o acréscimo de $\mathrm{PbO}$ devido a substituição do $\mathrm{Pb}^{2+}$ (diamagnético) por $\mathrm{Cu}^{2+}$ (paramagnético) [13].

Tabela 3: Valores da temperatura de Curie para amostras sinterizadas a $1000{ }^{\circ} \mathrm{C}$ por $6 \mathrm{~h}$.

\begin{tabular}{c|c}
\hline AMOSTRAS & Tc $\left(^{\circ} \mathbf{C}\right)$ \\
\hline$x=0,00$ & 493,00 \\
\hline$x=0,05$ & 480,00 \\
\hline$x=0,10$ & 474,00 \\
\hline$x=0,15$ & 473,00 \\
\hline$x=0,20$ & 469,00 \\
\hline
\end{tabular}


A influência da concentração de chumbo sobre as características físicas, densidade a verde (Dv), densidade aparente $(\mathrm{Da})$ e porosidade aparente $(\mathrm{Pa})$ e de suas respectivas densidades relativas das amostras sinterizadas a $1000{ }^{\circ} \mathrm{C} / 6$ h são apresentadas na Tabela 4.

Os valores da densidade a verde aumentaram de $2,31 \mathrm{~g} / \mathrm{cm}^{3}$ para a amostra isenta de chumbo $(\mathrm{x}=$ $0,00)$ para $2,88 \mathrm{~g} / \mathrm{cm}^{3}$ para a amostra com estequiometria contendo $\mathrm{x}=0,20$. O resultado (Dv/Dt) da amostra contendo $x=0,20$ foi superior a $50 \%$, o que mostra que houve uma boa densificação, exceto para a amostra isenta de chumbo $(\mathrm{x}=0,00)$ a amostra contendo $\mathrm{x}=0,15$. Após observou-se que a densidade aparente (Da) diminuiu de $5,00 \mathrm{~g} / \mathrm{cm}^{3}$ para a amostra isenta de chumbo $(\mathrm{x}=0,00)$ para $4,66 \mathrm{~g} / \mathrm{cm}^{3}$ para a amostra com estequiometria contendo $\mathrm{x}=0,20$. O decréscimo da densidade aparente com o acréscimo de PbO ocorreu devido ao aumento da espessura da camada da fase líquida rica em PbO localizada entre os grãos, consequentemente, a força de capilaridade entre os grãos diminuiu e consequentemente a densificação durante a sinterização desacelerou $[13,18,17]$.

Observou-se também que a relação entre os valores da densidade aparente com a densidade teórica (Da/Dt) diminuiu de 92,25 \% para a amostra isenta de chumbo ( $\mathrm{x}=0,00)$ para 86,45 \% para a amostra com estequiometria contendo $\mathrm{x}=0,20$ e seus resultados mostram que houve uma boa densificação durante a sinterização. Além disso, houve um aumento da porosidade na amostra isenta de chumbo $(x=0,00)$ de $1,62 \%$ para 9,41 \% na amostra com estequiometria contendo $x=0,05$. Para concentrações superiores a $x=0,05$ observou-se que não houve uma variação significativa da porosidade com o aumento da concentração de chumbo.

De uma maneira geral, o aumento da porosidade é um dos fatores que contribuíram para a diminuição da magnetização de saturação das amostras. A porosidade afeta o processo de magnetização porque os poros trabalham como gerador de um campo de desmagnetização, necessitando de um campo magnético muito alto para movimentar as paredes dos domínios [32,33,6].

Tabela 4: Características físicas das amostras sinterizadas a $1000{ }^{\circ} \mathrm{C} / 6 \mathrm{~h}$.

\begin{tabular}{|c|c|c|c|c|c|}
\hline \multirow{2}{*}{$\begin{array}{c}\text { AMOSTRAS } \\
\mathrm{x}\end{array}$} & \multicolumn{2}{|c|}{ AMOSTRAS NÃO SINTERIZADAS } & \multicolumn{3}{|c|}{ AMOSTRAS SINTERIZADAS } \\
\hline & $\operatorname{Dv}\left(\mathrm{g} / \mathrm{cm}^{3}\right)$ & Dv/Dt (\%) & $\mathrm{Da}\left(\mathrm{g} / \mathrm{cm}^{3}\right)$ & $\mathrm{Da} / \mathrm{Dt}(\%)$ & $\mathrm{Pa}(\%)$ \\
\hline 0,00 & 2,31 & 42,61 & 5,00 & 92,25 & 1,62 \\
\hline 0,05 & 2,62 & 48,61 & 4,93 & 91,46 & 9,41 \\
\hline 0,10 & 2,67 & 49,53 & 4,87 & 90,35 & 7,21 \\
\hline 0,15 & 2,67 & 49,53 & 4,66 & 86,45 & 6,42 \\
\hline 0,20 & 2,88 & 53,43 & 4,66 & 86,45 & 8,22 \\
\hline
\end{tabular}

(Densidade teórica (Dt): 5,39 $\mathrm{g} / \mathrm{cm}^{3}-J C P D S$ 34-0425)

(Densidade teórica (Dt): 5,42 g/cm³- JCPDS 01-077-0010)

A Tabela 5 mostra a variação do tamanho médio de grão para a amostra isenta de chumbo $(\mathrm{x}=0,00)$ a estequiometria contendo $\mathrm{x}=0,20$ sinterizadas a $1000^{\circ} \mathrm{C} / 6 \mathrm{~h}$.

Tabela 5: Tamanho médio de grão para as amostras com estequiometria contendo $\mathrm{x}=0,00 \mathrm{a} x=0,20$ sinterizadas a $1000^{\circ} \mathrm{C} / 6 \mathrm{~h}$.

\begin{tabular}{c|c}
\hline AMOSTRAS & $\begin{array}{c}\text { TAMANHO DE } \\
\text { GRÃO }(\mu \mathrm{m})\end{array}$ \\
\hline$x=0,00$ & $0,79 \pm 0,11$ \\
\hline$x=0,05$ & $1,03 \pm 0,21$ \\
\hline$x=0,10$ & $0,81 \pm 0,07$ \\
\hline$x=0,15$ & $1,04 \pm 0,05$ \\
\hline$x=0,20$ & $0,79 \pm 0,05$ \\
\hline
\end{tabular}


Analisando a Tabela 5 observa-se que não houve variação significativa do tamanho médio de grão da amostra isenta de chumbo $(\mathrm{x}=0,00)$ para a amostra com estequiometria contendo $\mathrm{x}=0,20$.

Nas amostras observou-se que a magnetização decresceu de 39,73 emu/g, na amostra isenta de chumbo $(\mathrm{x}=0,00)$, para 27,99 emu/g, na amostra com estequiometria contendo $\mathrm{x}=0,20$. A magnetização apresenta uma dependência com a característica extrínseca do material como o tamanho de grão e também com a característica intrínseca do material como a composição e a distribuição dos cátions na rede espinélio [6,23].

Neste caso, o decréscimo da magnetização de saturação de 39,73 emu/g, na amostra isenta de chumbo $(\mathrm{x}=0,00)$ para 27,99 emu/g, na amostra com estequiometria contendo $\mathrm{x}=0,20$ também é explicado devido que a característica intrínseca da composição nas amostras investigadas prevaleceu sobre a característica extrínseca, que no caso depende das variáveis de processamento, como é o caso do tamanho de grão [6,23].

As Figuras 4 a 8 mostram as micrografias da amostra isenta de chumbo $(x=0,00)$ a estequiometria contendo $\mathrm{x}=0,20$ sinterizadas a $1000^{\circ} \mathrm{C} / 6 \mathrm{~h}$. Nas micrografias observou-se que ocorreram a formação de fase líquida rica em $\mathrm{PbO}$ entre os grãos das amostras com estequiometria contendo $\mathrm{x}=0,05$ a estequiometria contendo $\mathrm{x}=0,20$. Neste caso observou-se que com o acréscimo de PbO acarretou ao aumento da espessura da camada da fase líquida rica em $\mathrm{PbO}$ diminuiu a densidade aparente das amostras [34,35,13].
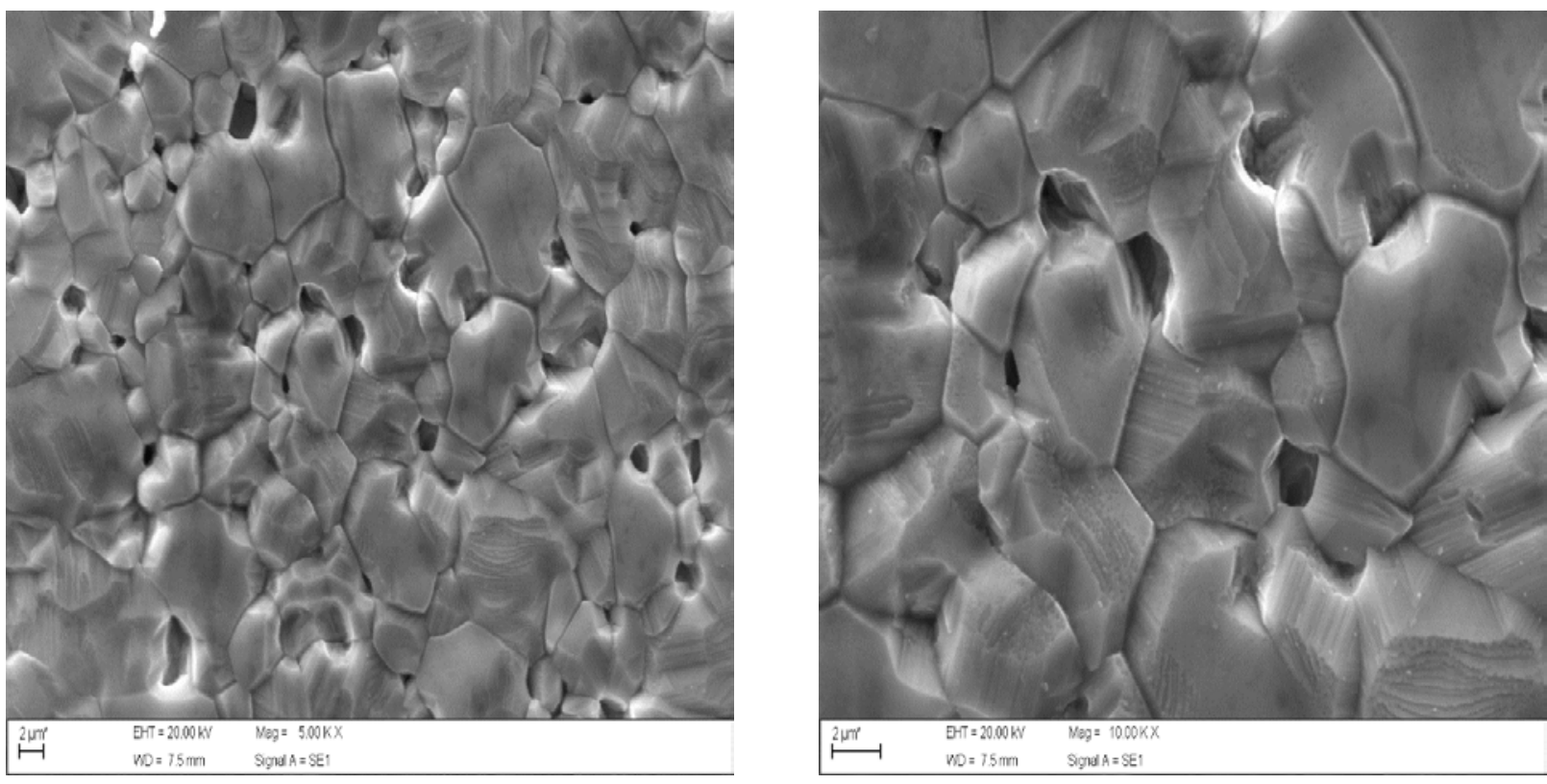

Figura 4: Microestrutura da amostra isenta de chumbo $(\mathrm{x}=0,00)$ sinterizada a $1000{ }^{\circ} \mathrm{C} / 6 \mathrm{~h}$. 

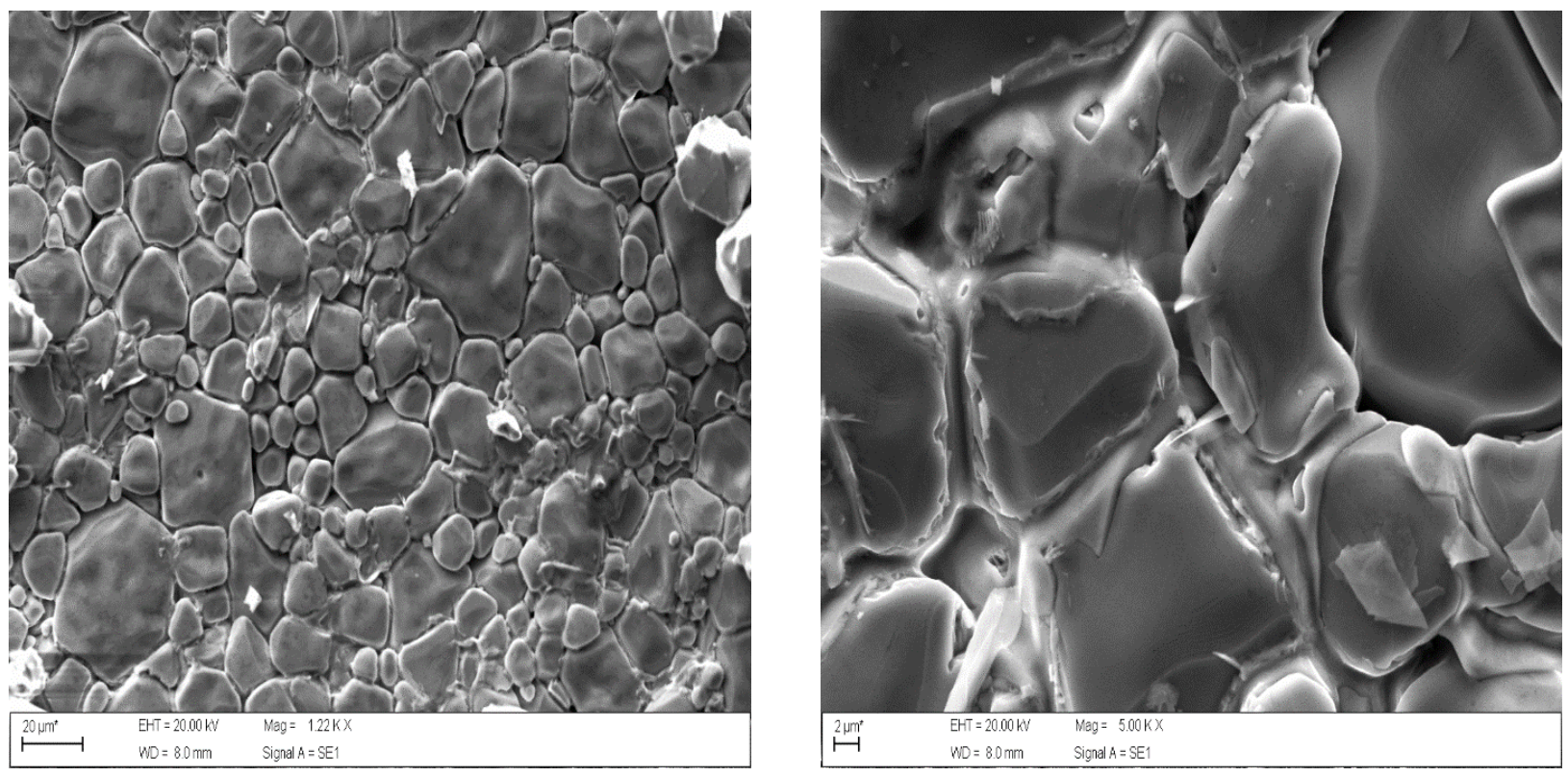

Figura 5: Microestrutura da amostra com estequiometria contendo $\mathrm{x}=0,05$ sinterizada a $1000{ }^{\circ} \mathrm{C} / 6 \mathrm{~h}$.
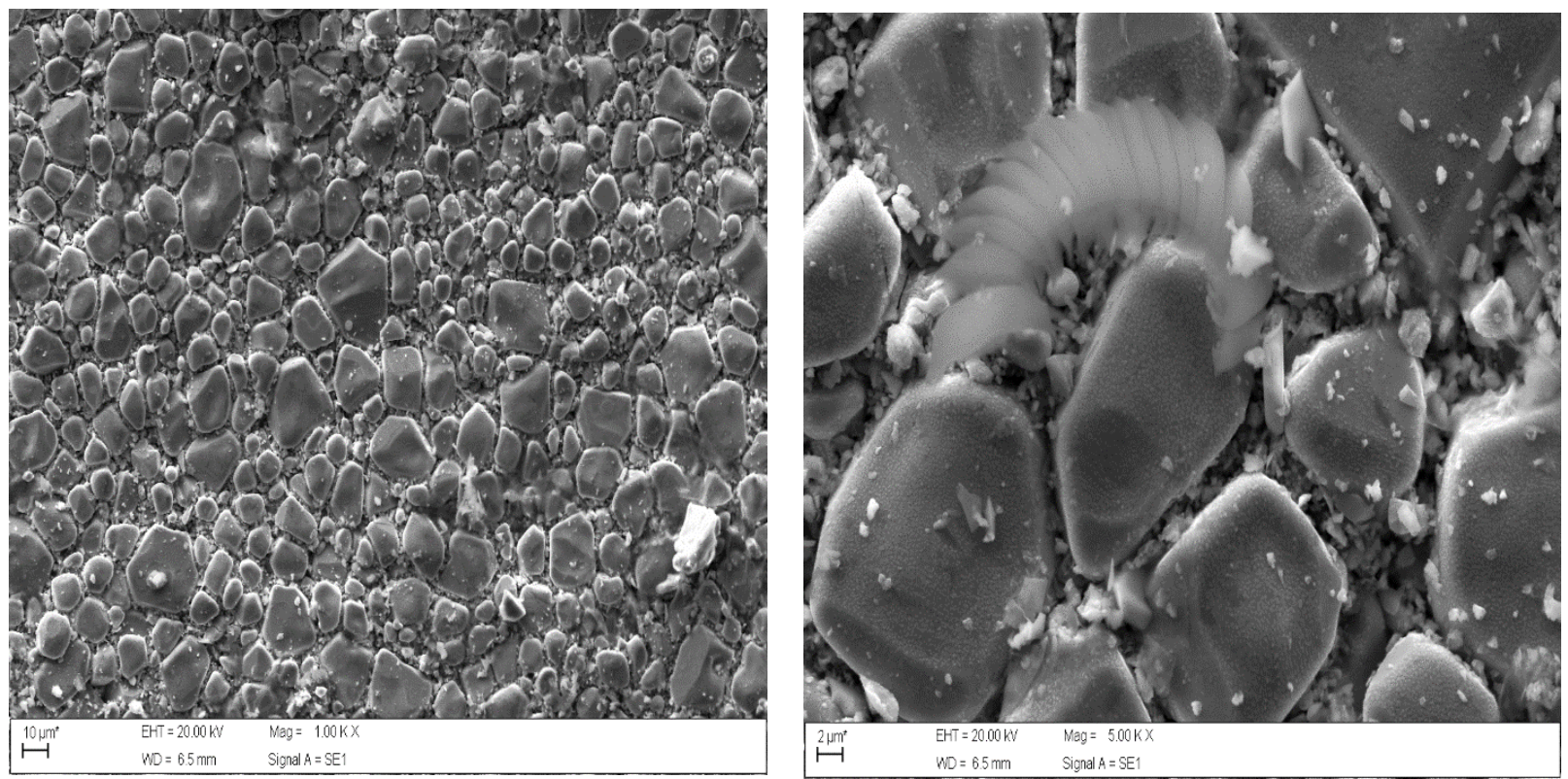

Figura 6: Microestrutura da amostra com estequiometria contendo $\mathrm{x}=0,10$ sinterizada a $1000{ }^{\circ} \mathrm{C} / 6 \mathrm{~h}$. 

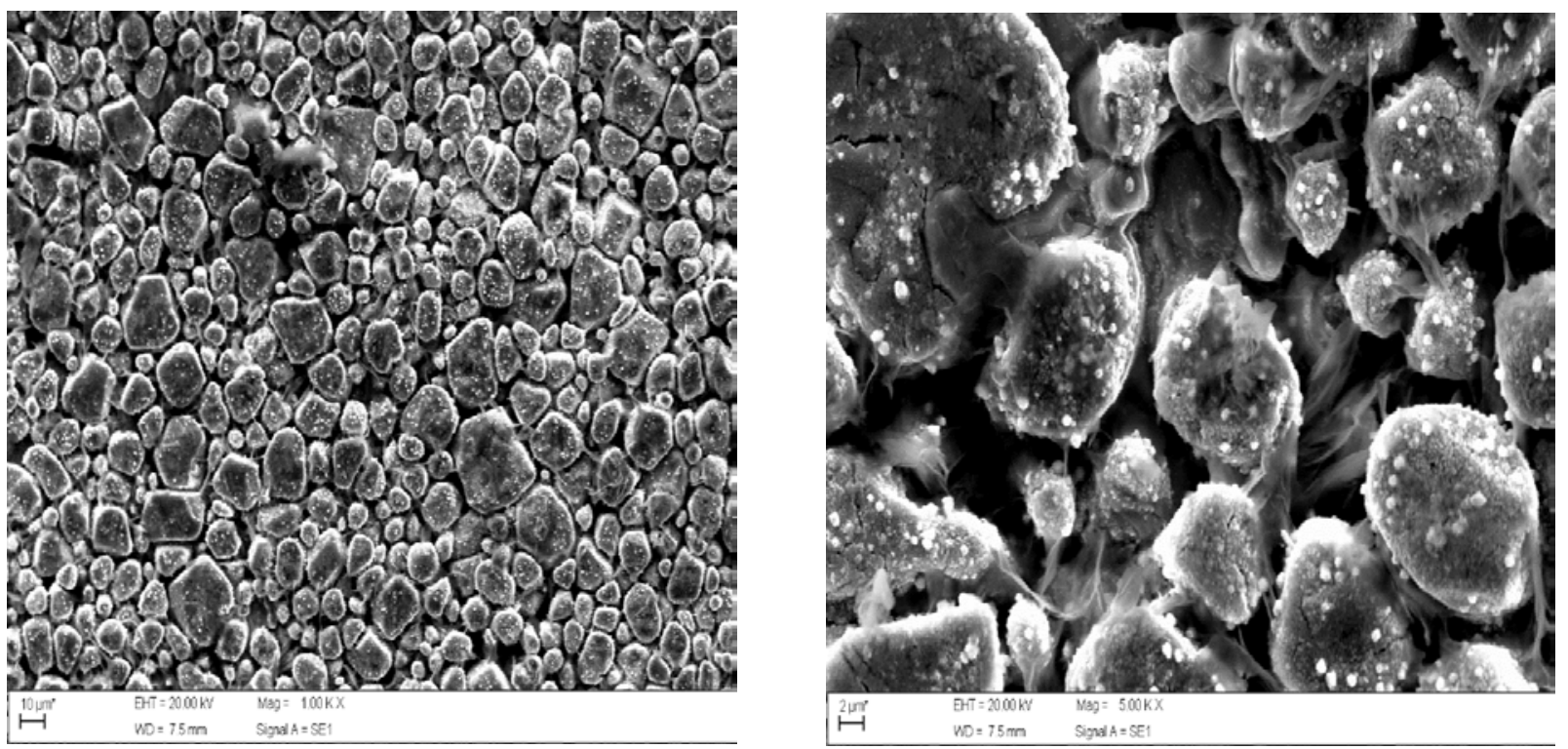

Figura 7: Microestrutura da amostra com estequiometria contendo $\mathrm{x}=0,15$ sinterizada a $1000{ }^{\circ} \mathrm{C} / 6 \mathrm{~h}$.
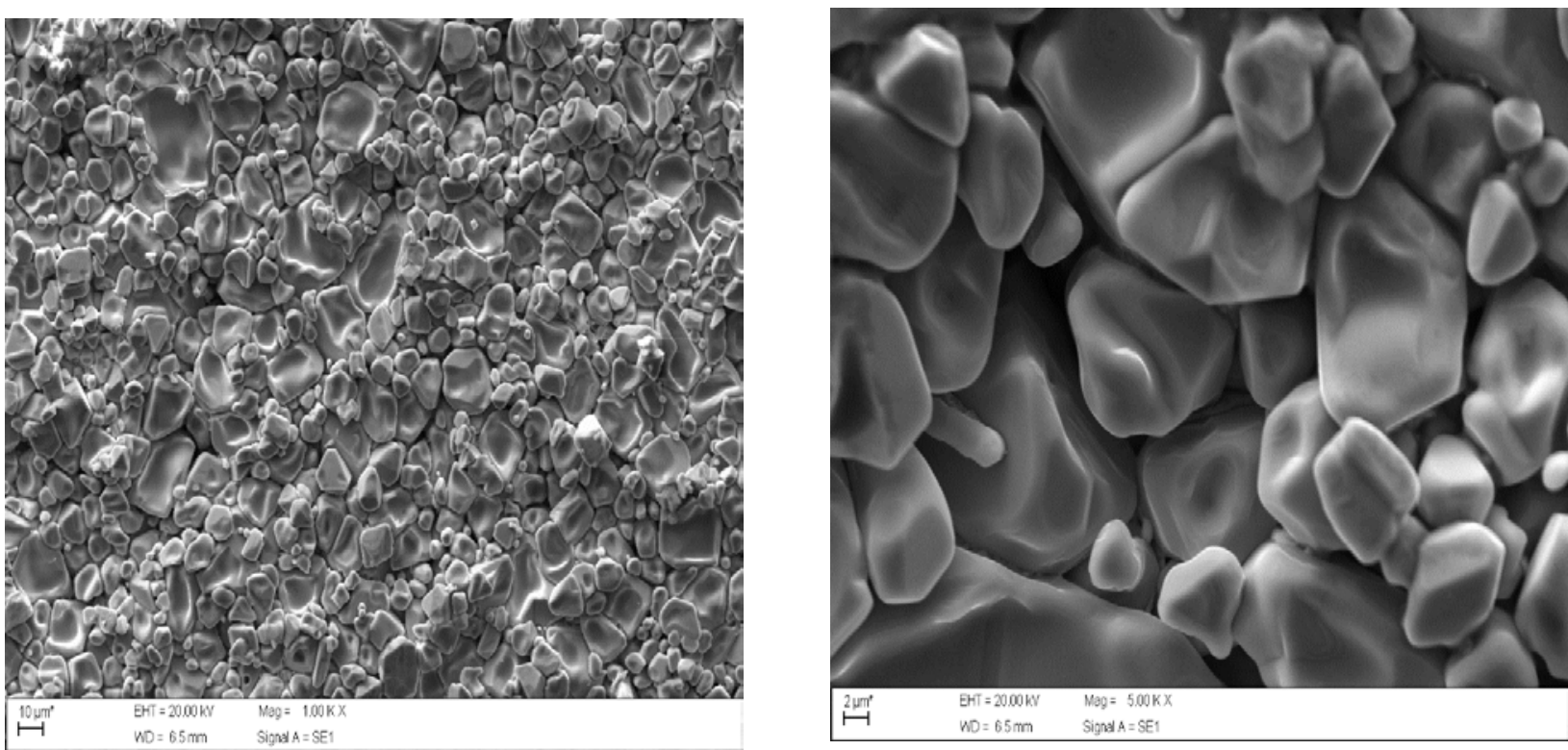

Figura 8: Microestrutura da amostra com estequiometria contendo com $\mathrm{x}=0,20$ sinterizada a $1000{ }^{\circ} \mathrm{C} / 6 \mathrm{~h}$.

\section{CONCLUSÕES}

Os resultados mostraram que a amostra com $\mathrm{x}=0,00$ é formada exclusivamente pela fase com a estrutura de espinélio, com simetria cúbica, já as amostras com estequiometria acima de $\mathrm{x}=0,05$ são formadas exclusivamente pela fase com a estrutura de espinélio com simetria tetragonal. O aumento da concentração de PbO proporcionou um aumento da porosidade devido ao aumento da espessura da camada da fase líquida rica em PbO localizada entre os grãos diminuindo a densidade aparente das amostras.

As curvas de histerese possuem características de um material magnético mole. Observou-se também que dois fatores contribuíram para a diminuição da magnetização de saturação com a concentração de chumbo: i) o aumento da porosidade e a ii) composição do material. Entretanto, observou-se que o campo coercivo aumentou com a concentração de chumbo devido a anisotropia magnetocristalina. 


\section{AGRADECIMENTOS}

Os autores agradecem a CAPES, CNPq e FAPEMIG pelos apoios financeiros.

\section{BIBLIOGRAFIA}

[1] SMIT, J., WIJN, H.P.J. Ferrites-Physical properties of Ferrimagnetic Oxides in Relation to Their Technical Applications. Eindhoven, Holland, N.V. Philips Gloeilampenfabrieken, 1959.

[2] SRIDHAR, R., RAVINDER D., KUMAR, K. V. "Synthesis and Characterization of Copper Substituted Nickel Nano-Ferrites by Citrate-Gel Technique", Advances in Materials Physics and Chemistry, v. 2, n. 3, pp. 192-199, 2012.

[3] HOQUE, S. M. ,ULLAH, M. S., KHAN, F.A.,et al. "Structural and magnetic properties of Li-Cu mixed spinel ferrites", Physica B, v. 406, n. 9, pp. 1799 - 1804, 2011.

[4] DUNITZ, J. D., ORGEL, L. E. "Eletronic proprietes of transition metal oxides: Distortions from cubic symmetry", J. Phys. Chem. Solids, v. 3, n. 1-2, pp. 20-29, 1957.

[5] DARUL, J. "Thermal instability of the tetragonally distorted structure of copper-iron materials", Z.Kristallogr.Suppl., v. 30, pp. 335-340, 2009.

[6] TORquATO, R. A., PORTElA, F. A., GAMA, L, et al. "Avaliação da microestrutura e das propriedades magnéticas de ferritas Ni-Zn dopadas com cobre", Cerâmica, v. 54, n. 329, pp. 55-62, 2008.

[7] KULKARNI, R.G., PATIL, V.U. "Jahn-Teller-type crystal distortions in copper ferrite", Journal of Materials Science, v. 15, n. 9, pp. 2221-2223, 1980.

[8] ATA-ALLAH, S. S., HASHHASH, A. "Jahn-Teller effect and superparamagnetism in zn substituted copper-gallate ferrite", Journal of Magnetism and Magnetic Materials, v. 307, n. 2, pp. 191-197, 2006.

[9] XIAO, Z., JIN, S., WANG, X., et al. "Preparation, structure and catalytic properties of magnetically separable Cu-Fe catalysts for glycerol hydrogenolysis", J. Mater. Chem, v. 12, n. 32, pp. 16598-16605, 2012.

[10] YANG, H., YAN , J., LU , Z., et al. "Photocatalytic activity evaluation of tetragonal CuFe2O4 nanoparticles for the $\mathrm{H}_{2}$ evolution under visible light irradiation", Journal of Alloys and Compounds, v. 476, n. 1-2, pp. 715-719, 2009.

[11] ZHANG, P., YU, B., ZHANG, L. "Mechanism of oxygen releasing of copper ferrite in the formation of the corresponding oxygen-deficient compound", Science in China Series B, v. 52, n. 1, pp. 101-108, 2009.

[12] PLOCEK, J., HUTLOVÁ, A., NIžňANSKý, D., et al. "Preparation of $\mathrm{CuFe}_{2} \mathrm{O} 4 / \mathrm{SiO}_{2}$ nanocomposite by the sol-gel method", Materials Science-Poland, v. 23, n. 3, pp. 697, 2005.

[13] MIRZAEE, O. "Influence of $\mathrm{PbO}$ and $\mathrm{TiO}_{2}$ additives on the microstructure development and magnetic properties of Ni-Zn soft ferrites", Journal of King Saud University - Engineering Sciences, v. 26, n. 2, pp. 152-158, 2013.

[14] SACHELARIE, L., DOROFTEI, C., REZLESCU, E. "Microstructure and magnetic and electrical properties of low-temperature sintering Ni-Zn, Li-Zn and Mg-Zn-Cu ferrites without and with addition of lead oxide", Journal of optoelectronics and advanced materials, v. 2, n. 4, pp. 864 - 867, 2010.

[15] GERMAN, R.M. Liquid Phase Sintering. New York, Springer Science \& Business, v. 2, 1985.

[16] VIEIRA ,D. C., BALAN, A. M. O. A., DURAN, R. M. "Evolução microestrutural da cerâmica $\mathrm{KSr}_{2} \mathrm{Nb}_{5} \mathrm{O}_{15}$ dopada com CuO: $\mathrm{B}_{2} \mathrm{O}_{3 "}$, Cerâmica, v. 55, n. 334, pp. 128-134, 2009.

[17] SILVA, A. G. P., JÚNIOR, C. A. "A sinterização rápida: sua aplicação, análise e relação com as técnicas inovadoras de sinterização", Cerâmica, v. 44, n. 290, pp. 225-232, 1998.

[18] REZLESCU, N., SACHELARIE , L., REZLESCU , E., et al. "Influence of PbO on microstructure and properties of a NiZn ferrite", Ceramics International, v. 29, n. 1, pp. 107-111, 2003.

[19] ULLAH, Z., ATIQ, S., NASEEM, S. "Influence of Pb doping on structural, electrical and magnetic 
properties of Sr-hexaferrites", Journal of Alloys and Compounds, v. 555, n. 5, pp. 263-267, 2013.

[20] HAQ, A., ANIS-UR-REHMAN, M. "Effect of Pb on structural and magnetic properties of Bahexaferrite", Physica B, v. 407, n. 5, pp. 822-826, 2012.

[21] ASTM STANDARD E 1382, 1997. Standard Test Methods for Determining Average Grain Size Using Semiautomatic and Automatic Image Analysis. ASTM International. West Conshohocken. 1997.

[22] MARCOMINI,R. F., SOUZA, D. M. P. F. "Caracterização microestrutural de materiais cerâmicos utilizando o programa de processamento digital de imagens "Image J", Cerâmica, n.57, pp.100-105, 2011.

[23] COSTA, A. C. F. M., MORELli; M. R., KIMINAMI, R. H. G. A. Microestrutura e propriedades magnéticas de ferritas Ni-Zn-Sm. Cerâmica, v. 49, n. 311, p. 168-173, 2003.

[24] DINIZ, V. C. S. , KIMINAMI, R. H. G. A., CORNEJO, D. R., et al. Estudo microestrutral e magnético de ferritas $\mathrm{Ni}_{0,5} \mathrm{Zn}_{0,5} \mathrm{Fe}_{2} \mathrm{O}_{4}$ sinterizadas por energia de micro-ondas, Revista Eletrônica de Materiais $e$ Processos, v. 9, n. 3, pp. 157-161, 2014.

[25] ABNT NBR 6220:2011. Materiais refratários densos conformados - Determinação do volume aparente, densidade de massa aparente, porosidade aparente, absorção e densidade aparente da parte sólida. São paulo, p. 4. 2011.

[26] MARIA, K. H., CHOUDHURY, S. , HAKIM, M. A. "Structural phase transformation and hysteresis behavior of Cu-Zn ferrites”, International Nano Letters, v. 42, pp. 1-10, 2013.

[27] SMITHA, P., PANDEY, P.K, KURIAN, S., et al., "Mossbauer studies and magnetic properties of spinel lead ferrite”, Hyperfine Interact, v. 184, n. 1-3, pp. 129-134, 2008.

[28] GAIKWAD , V. B., GAIKWAD, S. S., BORHADE, A. V., et al. "Synthesis and Characterization of Nano-Crystalline Cu and Pb”, Sensors \& Transducers, v. 134, n. 11, pp. 132-142, 2011.

[29] ROUXINOL, F. P. M. Propriedades Magnéticas de Filmes de Ligas Gd-Cr. Tese de D.Sc., Unicamp, Campinas, São Paulo, SP, Brasil, 2008.

[30] BALAGUROV, A. M., BOBRIKOV, I. A., MASCHENKO,M. S., et al. Structural phase transition in $\mathrm{CuFe}_{2} \mathrm{O}_{4}$ spinel. Crystallography Reports, v. 58, p. 696-703, 2013.

[31] AKHTER, S., PAUL , D. P., HAKIM , MD. A., et al. "Magnetic Properties of $\mathrm{Cu}_{1-\mathrm{x}} \mathrm{Zn}_{\mathrm{x}} \mathrm{Fe}_{2} \mathrm{O}_{4}$ Ferrites with the Variation of Zinc Concentration", Journal of Modern Physics, v. 3, pp. 398-403, 2013.

[32] RAMKRISHNA, H. K., ASIF, K., ANKUSH, B. K., et al. "Influence of $\mathrm{Cr}^{3+}$ substitution on the electrical and magnetic properties of $\mathrm{Ni}_{0.4} \mathrm{Cu}_{0.4} \mathrm{Zn}_{0.2} \mathrm{Fe}_{2} \mathrm{O} 4$ nanoparticles", International Nano Letters, $\mathrm{v}$. 28, n. 2, pp. 1-5, 2012.

[33] CHAUDHARI, M.V., SHIRSATH, SAGAR E., et al., "Site occupancies of Co-Mg-Cr-Fe ions and their impact on the properties of $\mathrm{Co}_{0.5} \mathrm{Mg}_{0.5} \mathrm{Cr}_{\mathrm{x}} \mathrm{Fe}_{2-\mathrm{x}} \mathrm{O}_{4}$ ", Journal of Alloys and Compounds, v. 552, pp. 443-450, 2013.

[34] GERMAN, R. M., SURI, P., PARK, S. J. "Review: liquid phase sintering", Journal of Materials Science, v. 44, pp. 1-39, 2009.

[35] MARCHI, J. Sinterizaçâo via fase líquida de cerâmicas â base de carbeto de silício com aditivos óxidos utilizando planejamento de experimentos. Tese de D.Sc., IPEN. São Paulo,SP, Brasil, 2003. 